\title{
The Young and the Massive: Stars at the upper end of the Initial Mass Function
}

\author{
Saida M. Caballero-Nieves ${ }^{1,2}$ and P. A. Crowther ${ }^{2}$ \\ ${ }^{1}$ Department of Physics \& Space Sciences, Florida Institute of Technology \\ 150 West University Blvd, Melbourne, FL 32901, USA \\ email: scaballero@fit.edu \\ ${ }^{2}$ Dept. of Physics \& Astronomy, University of Sheffield, Hicks Building, Hounsfield RD \\ Sheffield S3 7RH, United Kingdom
}

\begin{abstract}
The upper mass limit of stars remains an open question in astrophysics. Here we discuss observations of the most massive stars (greater than 100 solar masses) in the local universe and how the observations fit in with theoretical predictions. In particular, the Large Magellanic Cloud plays host to numerous very massive stars, making it an ideal template to study the roles that environment, metallicity, and multiplicity play in the formation and evolution of the most massive stars. We will discuss the work that is instrumental in laying the groundwork for interpreting future observations by James Webb of starburst regions in the high redshift universe.
\end{abstract}

Keywords. stars: early-type, stars: mass function, stars: fundamental parameters (masses)

\section{Introduction to Very Massive Stars}

Very Massive Stars (VMS) are defined as stars born at the upper end of the Initial Mass Function (IMF). They have initial masses, $M_{i}$, greater than $100 M_{\odot}$. These stars are very rare but play an important role over a large range of scales in our Universe. Their strong stellar winds along with their subsequent supernova enrich the interstellar medium and contribute to the chemical evolution of galaxies. In the high redshift Universe, very massive stars dominate the light in unresolved clusters of starburst galaxies. Therefore, they play a crucial role in creating population synthesis models to fit the observations.

VMS provide an interesting observational challenge. The most common stellar population synthesis (Leitherer et al. 1999) and stellar evolutionary codes (Meynet \& Maeder 2000) do not include stars with initial masses exceeding 100 or $120 M_{\odot}$, respectively. This introduces inconsistencies in modelling starburst regions in the distant universe. In this paper, we discuss the observational evidence for stars that exceed $100 M_{\odot}$ and make the case for the need to include such stars in future population synthesis codes.

For further reading, we recommend Martins (2015), an excellent review on the observational properties of VMS, Zinnecker \& Yorke (2007), which remains a seminal review on the formation of massive stars, and references therein.

\section{VMS in the Milky Way}

The most obvious place to find VMS is at the core of young, dense and massive clusters. Weidner \& Kroupa (2006) concluded from observations that there exists a well-defined relation between the most massive star in a cluster and the cluster mass. This means that the more massive a cluster, the more massive stars you can form. In addition, one needs to observe these clusters at early stages $(\lesssim 3 \mathrm{Myr})$ to successfully catch the most 
massive members before they evolve. In this section we provide highlights of Galactic VMS stars in clusters and in some cases, more isolated regions.

\subsection{VMS in Cluster cores}

The starburst cluster NGC 3603 is a HII region, rich in high mass stars. The very young age (1-2 Myr; Melena et al. 2008), based on the presence of hydrogen-rich and nitrogenrich main sequence Wolf-Rayet stars (WNh) along with a young O star population, makes it an ideal cluster to search for VMS. It contains two VMS in the core: A1 and B. In the case of A1, it is a binary where the two components have masses of $116 M_{\odot}+89 M_{\odot}$ (Schnurr et al. 2008) and are among the most massive stars dynamically weighed.

The massive open cluster, Arches Cluster, was only discovered in the mid-nineties (Cotera 1995, Figer 1995, Nagata et al. 1995) due to high foreground extinction towards the Galactic Center. Based on the cluster mass estimated of $\sim 4-6 \times 10^{4} M_{\odot}$ estimated by Figer (2005)), the Arches cluster has a high enough mass content to form VMS. They also determined from the IMF an upper mass limit cutoff of $150 M_{\odot}$. Oey \& Clarke $(2005)$ arrived to a similar conclusion using a statistical approach with a sample of clusters and OB associations. However, both of these results are limited by the extinction laws used (as is the case for the Arches Cluster) and the slope of the initial mass function (IMF) for stars greater than $10 M_{\odot}$. In this high mass range, radiation pressure dominates with a shallower mass-luminosity relationship, such that for $\mathrm{L} \propto \mathrm{M}^{\alpha}, \alpha$ tends towards unity as $\mathrm{M}$ tends towards infinity. Zero age main sequence VMS approach the Eddington limit, but do not initially exceed it.

Like NGC 3603, the most massive members of the Arches cluster are found in its core. The spectroscopy study by Martins et al. (2008) estimated that the WNh population in the core of the Arches were around $120 M_{\odot}$. The revised interpretation by Crowther et al. 2010 using AO-based VLT photometry, found that two stars, F6 and F9, have initial masses of $185 M_{\odot}$ and $165 M_{\odot}$, respectively, exceeding the $150 M_{\odot}$ upper limit. Schneider et al. (2014) attribute the WNh stars to binary stellar mergers, giving an older age estimate of $\sim 3.5 \mathrm{Myr}$ than that of NGC 3603. The complicated nature of the Arches Cluster do not make it the ideal candidate to study the stellar upper mass limit cut off.

\subsection{VMS in large star forming regions}

The prior two cases we looked at considered VMS in cluster environments. Here we note at least two cases of known VMS stars outside density cluster cores in intermediate dense regions of major massive star forming regions. This implies that you can make stars with $\mathrm{M}_{i n i}>100 M_{\odot}$ outside dense cluster cores.

One of the most enigmatic stars in the Milky Way is $\eta$ Carinae, a Luminous Blue Variable (LBV). It has gone through two eruptive mass loss events in the last two hundred years. It is believed to have $\mathrm{M}_{\text {current }} \sim 120 M_{\odot}$ (Hillier et al. 2010 ), but lost $\sim 20 M_{\odot}$ in the last 170 years (Morris et al. 1999), falling into the category of VMS. It is also part of a binary system that is likely to play a role in the eruption events. $\eta$ Car is usually associated with the dense star cluster Trumpler 16. However, unlike the VMS discussed previously typically found in the cluster center, it sits on the periphery of the cluster. This interesting star is discussed further in this proceedings by A. Damineli, K. Hamaguchi, M. Kiminki, T. Madura, S. Owocki and N. Smith.

In the nearby OB association, Cyg OB2, dwells a peculiar B hypergiant. Thought to be a quiescent LBV or a precursor to an LBV, Cyg OB2-12 is estimated to be a $120 M_{\odot}$ star (Clark et al. 2012), with a high X-ray luminosity indicating that it is in a colliding wind system. The history of the association from dynamical studies (Wright et al. 2014) and the lack of mass segregation (Parker \& Wright 2016), indicates that Cyg OB2 has 


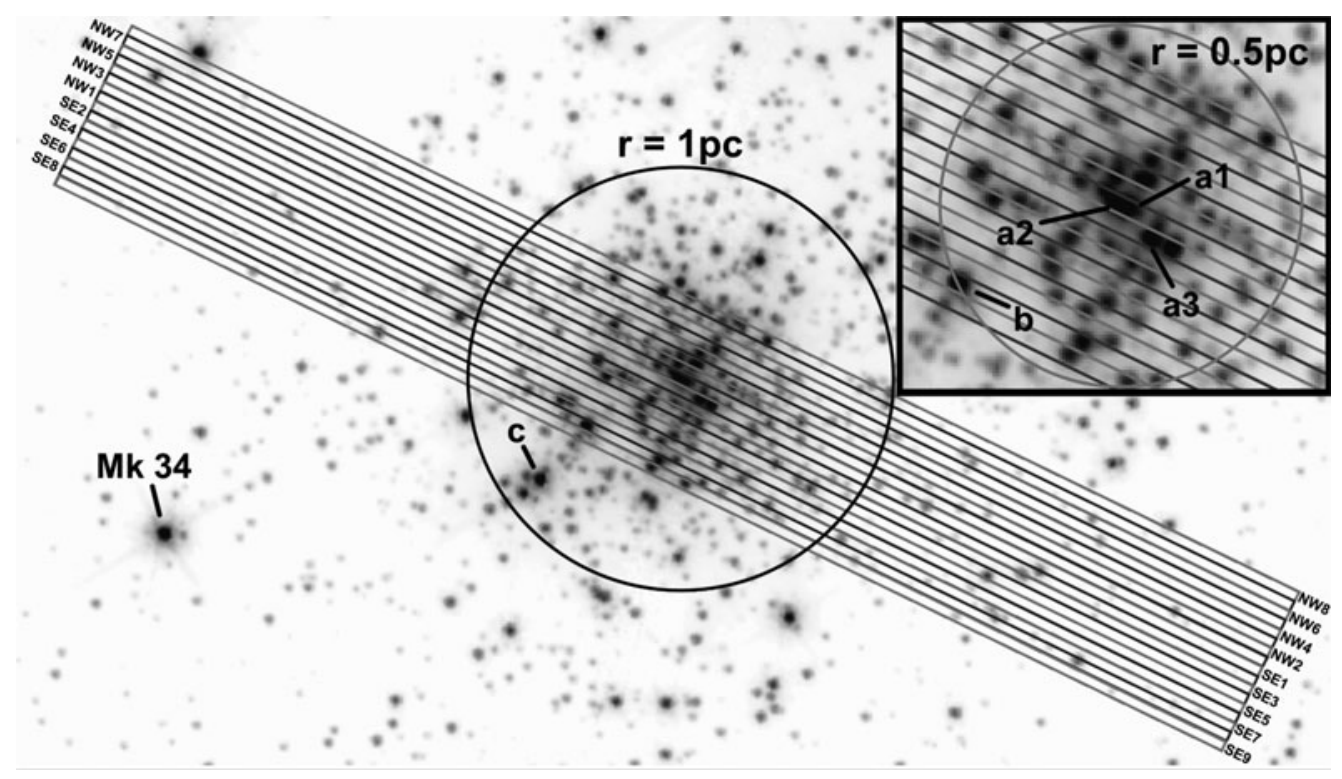

Figure 1. HST/STIS slits $(52 " \times 0 " .2)$ superimposed upon an F336W WFC3/UVIS image of R136 (de Marchi et al. 2011), oriented with North up and East to left, together with a circle of radius 4."1 (1 parsec) and identification of the acquisition star Melnick 34. The active slit length for MAMA observations is the central 25". The zoom highlights the central region, including identification of individual slits and the integrated R136a cluster (2." 05 radius circle, centered upon R136a1 equivalent to 0.5 parsec at the distance of the LMC). Adapted from Crowther et al. (2016).

always been a relatively low density environment. Thus, high densities are not necessary to form VMS, but make it more likely to find VMS.

\section{VMS in the LMC: R136}

The star forming region 30 Doradus, or the Tarantula nebula, in the Large Magellanic Cloud (LMC) is the brightest star forming region in the Local Group. It is rich in VMS with $\sim 20$ stars scattered throughout the region (Table 8; Crowther et al. 2016). The region is a conglomeration of different bursts of star formation and the stellar content cannot be treated as being coeval.

At the core of the nebula sits the starburst cluster R136, once thought to be a supermassive star $\left(2,500 M_{\odot}\right.$; Cassinelli et al. 1981). It was finally resolved as a cluster with interferometry (Weigelt et al. 1985) and Hubble imaging (Hunter et al. 1995). R136 is ideally situated compared to Milky Way clusters, with little extinction along the line of sight and resolvable. The core is the source of feedback to the larger region through the ultraviolet (UV) radiation of its most massive members. It serves as a resolved template of starburst regions in distant galaxies.

The central parsec of R136 has a long standing history of being difficult to resolve its individual members. However it is crucial to understand both the integrated properties of the cluster itself and the individual members. R136 contains several VMS stars, among them the brightest is thought to be the most massive star known, R136a1 ( $M_{i n i} \sim$ $300 M_{\odot}$; Crowther et al. 2010). The mass of the R136a1 is still a subject of uncertainty (see M. M. Díez, this proceedings), and hence the stellar upper mass limit remains an open question. 


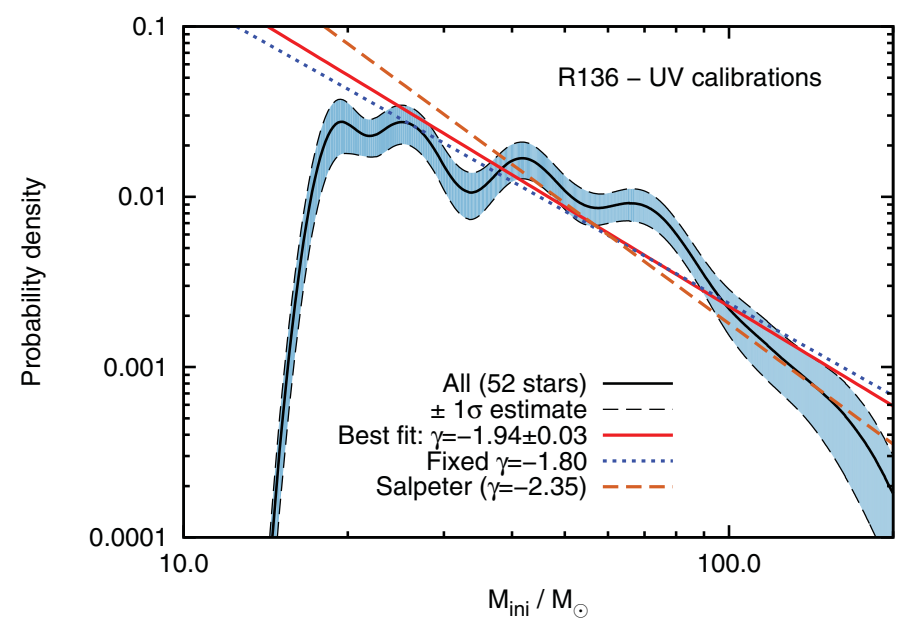

Figure 2. Initial mass function based on the UV derived parameters for the central stars in R136 using the bayesian tool BONNSAI (Schneider et al. 2014). The best fit IMF of R136 has a shallower slope for the most massive stars than the standard Salpeter slope. (F. Schneider, private communication).

Crowther et al. (2016) use Hubble STIS to create an Integral Field Spectrum (IFS) of the entire central parsec to determine both the individual properties of the stellar content along with the combined cluster properties (see Figure 1). Here we discuss the results and some of the implications from this study.

Massive stars are well known to love company, and are often found with at least one companion that will influence their evolution (Sana et al. 2012). In preliminary results of the multiplicity of the O-star sample in R136, we find that about $35 \%$ of them show a significant radial velocity variation that could be due to a companion. It therefore follows that these VMS could be found in binary systems too. For mass estimates, we have to ascertain that we are accounting for the correct number of stars in the luminosity we measure. We consider specifically R136a1 and the possibility that it is a binary star at different scales. To really affect the mass estimates, we are looking for two near equal mass stars, or near equal brightness, that are very close together. Schnurr et al. (2009) carried out a spectroscopic study and determined that in the period range they considered, R136a1 appeared single. It showed no radial velocity variations that could be attributed to a companion with $\mathrm{P} \lesssim 44$ days. They found that $\mathrm{R} 136 \mathrm{c}$ did seem to show radial velocity variations. This is consistent with the high X-ray luminosity observed for this star.

The X-ray properties of the central stars in R136 can also indicate binarity. High Xray fluxes can be attributed to colliding winds, i.e. two stars with strong stellar winds causing a shock region where the winds meet, emitting X-rays. Crowther et al. (2010) found that the X-ray flux from the center of R136 precluded pairs of equal mass VMS in close binary orbits within a few hundred AU.

To explore the regions at wider separations where the colliding winds will not create a bright X-ray shock region or show an radial velocity variability, we need high spatial resolution observations. Hubble's interfertometer, the Fine Guidance Sensor is the best instrument to further constrain the parameter space for determining binarity of these systems. Figure 3 shows the raw interferometric signal of the region, with each ' $\mathrm{S}$ ' 


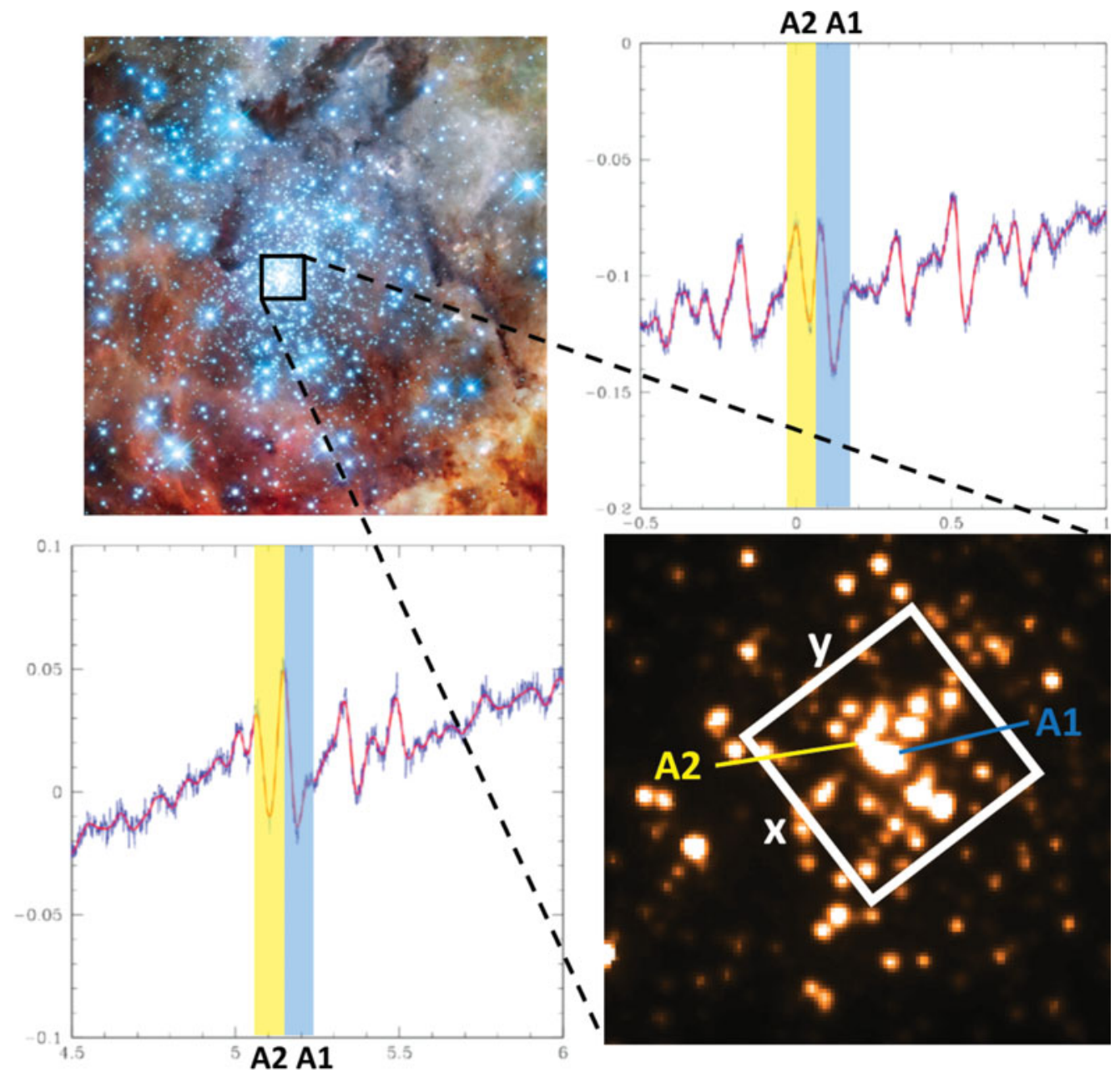

Figure 3. The super star cluster R136 at the center of 30 Doradus (top left). The cluster contains the 9 VMS, including R136a1 the most massive star known (bottom right). FGS scans in 2 perpendicular directions (top right \& bottom left) allows for an unprecedented close up of this dense region.

corresponding to a star in the field. The density of the region will make disentangling the signal for stars at the center of R136 exceptionally challenging.

Crowther et al. (2016) provide a complete census of the most massive stars in the core of R136. Figure 2 (F. Schneider, private communication) shows the initial mass function based on the UV derived parameters for the central stars in R136 using the bayesian tool BONNSAI (Schneider et al. 2014). This very preliminary result hints at the possibility that the IMF of R136 has a shallower slope for the most massive stars than the standard Salpeter slope.

\section{VMS in the high redshift Universe}

From Crowther et al. (2016) we find that a handful of VMS contribute significantly to the integrated UV spectrum of the entire cluster. In 30 Doradus, $\sim 30$ VMS contribute significantly to the total ionizing output (Doran et al. 2013). A third of those VMS are 
found in the center of R136. Of course, the rest-frame UV spectra of star forming galaxies in the early universe are redshifted to the near-infrared where JWST is exceptionally sensitive. Therefore, to interpret the rest-frame UV spectrum of distant starbursts, the presence of VMS needs to be considered (see Crowther et al., these proc). Indeed, some stellar population synthesis codes (e.g. BPASS; Stanway et al. 2016) now account for VMS and close binary evolution.

\section{References}

Cassinelli, J. P., Mathis, J. S., \& Savage, B. D. 1981, Science, 212, 1497

Clark, J. S., Najarro, F., Negueruela, I., et al. 2012, A\& $A p$, 541, A145

Cotera, A. S. 1995, Ph.D. Thesis

Crowther, P. A., Schnurr, O., Hirschi, R., et al. 2010, MNRAS, 408, 731

Crowther, P. A., Caballero-Nieves, S. M., Bostroem, K. A., et al. 2016, MNRAS, 458, 624

De Marchi, G., Paresce, F., Panagia, N., et al. 2011, ApJ, 739, 27

Doran, E. I., Crowther, P. A., de Koter, A., et al. 2013, A\& Ap, 558, A134

Figer, D. F. 1995, Ph.D. Thesis

Figer, D. F. 2005, Nature, 434, 192

Hillier, D. J., Davidson, K., Ishibashi, K., \& Gull, T. 2001, ApJ, 553, 837

Hunter, D. A., Shaya, E. J., Holtzman, J. A., et al. 1995, ApJ, 448, 179

Köhler, K., Langer, N., de Koter, A., et al. 2015, A\&Ap, 573, A71

Leitherer, C., Schaerer, D., Goldader, J. D., et al. 1999, ApJS, 123, 3

Martins, F., Hillier, D. J., Paumard, T., et al. 2008, A\& Ap, 478, 219

Martins, F. 2015, Very Massive Stars in the Local Universe, 412, 9

Melena, N. W., Massey, P., Morrell, N. I., \& Zangari, A. M. 2008, AJ, 135, 878

Meynet, G. \& Maeder, A. 2000, A\&Ap, 361, 101

Morris, P. W., Waters, L. B. F. M., Barlow, M. J., et al. 1999, Nature, 402, 502

Nagata, T., Woodward, C. E., Shure, M., \& Kobayashi, N. 1995, AJ, 109, 1676

Parker, R. J. \& Wright, N. J. 2016, MNRAS, 457, 3430

Oey, M. S. \& Clarke, C. J. 2005, ApJL, 620, L43

Sana, H., de Mink, S. E., de Koter, A., et al. 2012, Science, 337, 444

Schneider, F. R. N., Izzard, R. G., de Mink, S. E., et al. 2014, ApJ, 780, 117

Schneider, F. R. N., Langer, N., de Koter, A., et al. 2014, A\&A Ap, 570, A66

Schnurr, O., Casoli, J., Chené, A.-N., Moffat, A. F. J., \& St-Louis, N. 2008, MNRAS, 389, L38

Schnurr, O., Chené, A.-N., Casoli, J., Moffat, A. F. J., \& St-Louis, N. 2009, MNRAS, 397, 2049

Stanway, E. R., Eldridge, J. J., \& Becker, G. D. 2016, MNRAS, 456, 485

Weigelt, G. \& Baier, G. 1985, A\&Ap, 150, L18

Weidner, C. \& Kroupa, P. 2006, MNRAS, 365, 1333

Wright, N. J., Parker, R. J., Goodwin, S. P., \& Drake, J. J. 2014, MNRAS, 438, 639

Yusof, N., Hirschi, R., Meynet, G., et al. 2013, MNRAS, 433, 1114

Zinnecker, H. \& Yorke, H. W. 2007, ARAA, 45, 481 